\title{
Robust and Clean Majorana Zero Mode in the Vortex Core of High-Temperature Superconductor $\left(\mathrm{Li}_{0.84} \mathrm{Fe}_{0.16}\right) \mathrm{OHFeSe}$
}

\author{
Qin Liu, ${ }^{1,2,3}$ Chen Chen, ${ }^{1,3}$ Tong Zhang, ${ }^{1,3,{ }^{*}}$ Rui Peng, ${ }^{1,3}$ Ya-Jun Yan, ${ }^{1,3}$ Chen-Hao-Ping Wen, ${ }^{1,3}$ Xia Lou, ${ }^{1,3}$ \\ Yu-Long Huang, ${ }^{4}$ Jin-Peng Tian, ${ }^{4}$ Xiao-Li Dong, ${ }^{4}$ Guang-Wei Wang, ${ }^{5}$ Wei-Cheng Bao, ${ }^{6,7}$ Qiang-Hua Wang, ${ }^{3,6}$ \\ Zhi-Ping Yin, ${ }^{5, \dagger}$ Zhong-Xian Zhao, ${ }^{4}$ and Dong-Lai Feng ${ }^{1,3, \hbar}$ \\ ${ }^{1}$ State Key Laboratory of Surface Physics, Department of Physics, \\ and Advanced Materials Laboratory, Fudan University, Shanghai 200438, China \\ ${ }^{2}$ Science and Technology on Surface Physics and Chemistry Laboratory, \\ Mianyang, Sichuan 621908, China \\ ${ }^{3}$ Collaborative Innovation Center of Advanced Microstructures, Nanjing 210093, China \\ ${ }^{4}$ Beijing National Laboratory for Condensed Matter Physics and Institute of Physics, \\ Chinese Academy of Sciences, Beijing 100190, China \\ ${ }^{5}$ Department of Physics and Center for Advanced Quantum Studies, \\ Beijing Normal University, Beijing 100875, China \\ ${ }^{6}$ National Laboratory of Solid State Microstructures \& School of Physics, \\ Nanjing University, Nanjing, 210093, China \\ ${ }^{7}$ Zhejiang University of Water Resources and Electric Power, Hangzhou 310018, China
}

(Received 27 July 2018; published 28 December 2018)

\begin{abstract}
The Majorana fermion, which is its own antiparticle and obeys non-Abelian statistics, plays a critical role in topological quantum computing. It can be realized as a bound state at zero energy, called a Majorana zero mode (MZM), in the vortex core of a topological superconductor, or at the ends of a nanowire when both superconductivity and strong spin orbital coupling are present. A MZM can be detected as a zero-bias conductance peak (ZBCP) in tunneling spectroscopy. However, in practice, clean and robust MZMs have not been realized in the vortices of a superconductor because of contamination from impurity states or other closely packed Caroli-de Gennes-Matricon (CdGM) states, which hampers further manipulations of MZMs. Here, using scanning tunneling spectroscopy, we show that a ZBCP well separated from the other discrete CdGM states exists ubiquitously in the cores of free vortices in the defect-free regions of $\left(\mathrm{Li}_{0.84} \mathrm{Fe}_{0.16}\right) \mathrm{OHFeSe}$, which has a superconducting transition temperature of $42 \mathrm{~K}$. Moreover, a Diraccone-type surface state is observed by angle-resolved photoemission spectroscopy, and its topological nature is confirmed by band calculations. The observed ZBCP can naturally be attributed to a MZM arising from the chiral topological surface state of a bulk superconductor. Thus, $\left(\mathrm{Li}_{0.84} \mathrm{Fe}_{0.16}\right) \mathrm{OHFeSe}$ provides an ideal platform for studying MZMs and topological quantum computing.
\end{abstract}

DOI: 10.1103/PhysRevX.8.041056

Subject Areas: Condensed Matter Physics

\section{INTRODUCTION}

In recent years, many recipes have been proposed for realizing MZMs [1-3], as a critical step towards topological quantum computation [4,5]. For example, MZMs are predicted to exist at the ends of a semiconductor nanowire with strong spin-orbital coupling (SOC) when it is in

\footnotetext{
tzhang18@fudan.edu.cn

†yinzhiping@bnu.edu.cn

*dlfeng@fudan.edu.cn
}

Published by the American Physical Society under the terms of the Creative Commons Attribution 4.0 International license. Further distribution of this work must maintain attribution to the author(s) and the published article's title, journal citation, and DOI. proximity to a superconductor and under a sufficiently large Zeeman field [2,3,6-8]. A quantized ZBCP has been observed in the tunneling spectrum of a hybrid device between superconducting aluminum and an InSb nanowire [9], providing compelling evidence for a MZM. However, fundamental quantum computing operations, such as braiding of MZMs in nanowires [10], are challenging and yet to be realized after a tremendous amount of ingenious technical endeavors [11,12].

Topological systems with additional dimensions provide a broader hunting ground in the search of MZM, e.g., in the vortex cores of a topological superconductor or superconducting heterostructures [1-3]. For conventional $s$-wave superconductors, confined quasiparticles in the vortex core give rise to CdGM bound states with $E=$ $\mu \Delta^{2} / E_{F}$, where $\mu$ is a half integer $( \pm 1 / 2, \pm 3 / 2 \ldots)$ [13]. 
However, for a chiral $p$-wave superconductor, bound states still exist but with integer $\mu(0, \pm 1, \pm 2 \ldots)$-the $E=0$ state, or zero-energy mode, is a MZM [14]. Chiral $p$-wave superconductors are extremely scarce, but $\mathrm{Fu}$ and Kane have proposed that proximity effects from an $s$-wave superconductor on topological surface states would produce a two-dimensional system whose Hamiltonian effectively resembles a spinless $p \pm i p$ superconductor, and thus it can host Majorana bound states in its vortices [15] (the spinless nature is due to the fact that the topological surface state is spin nondegenerate). Based on this scenario, ZBCPs that may potentially correspond to MZMs have been found in the vortex cores of topological insulator-superconductor heterostructures (e.g., $\mathrm{Bi}_{2} \mathrm{Te}_{3} / \mathrm{NbSe}_{2}$ and $\mathrm{Bi}_{2} \mathrm{Te}_{3} / \mathrm{FeTe}_{x} \mathrm{Se}_{1-x}$ ) [16-18], and topological surface states of bulk superconductors (e.g., $\mathrm{Cu}_{x} \mathrm{Bi}_{2} \mathrm{Se}_{3}$ and $\mathrm{FeTe}_{x} \mathrm{Se}_{1-x}$ ) [19-22]. However, an unambiguous identification or isolation of a MZM is still lacking in these systems. For materials with a large Fermi energy $\left(E_{F}\right)$, such as $\mathrm{Bi}_{2} \mathrm{Se}_{3} / \mathrm{NbSe}_{2}, \mathrm{Cu}_{x} \mathrm{Bi}_{2} \mathrm{Se}_{3}$, and $\mathrm{Bi}_{2} \mathrm{Te}_{3} / \mathrm{FeTe}_{x} \mathrm{Se}_{1-x}$, a mode at zero energy is buried under many densely packed CdGM states in the vortex core, their energies separated by only a few $\mu \mathrm{eV}$. Here, $\mathrm{FeTe}_{x} \mathrm{Se}_{1-x}$ has a small $E_{F}$, so its ZBCP can be readily distinguished from the CdGM states. However, there are intrinsic impurity effects of interstitial $\mathrm{Fe}$ and heavy $\mathrm{Te}$ doping $[23,24]$. A zero-bias impurity state was observed on interstitial Fe, which is insensitive to the magnetic field [23], but whether this is related to a MZM is yet to be confirmed. A ZBCP can be observed in a fraction of vortices in certain $\mathrm{FeTe}_{x} \mathrm{Se}_{1-x}$ samples [21], while it cannot be observed in samples annealed to reduce impurities [24]. Therefore, to study the largely unknown properties of MZMs, one needs to find a system that can host MZMs in a clean and robust manner, with weak pinning and scattering from impurities, which would lay the foundation for further operations such as braiding of MZMs.

To reach these goals, we need a superconducting system that has a large superconducting gap, a small $E_{F}$, a stoichiometric lattice (at least in the sublattice responsible for superconductivity) with few defects, a short superconducting coherence length $(\xi)$ to potentially reduce vortex pinning, and a topologically nontrivial electronic structure. The large gap usually also means high superconducting transition temperature $\left(T_{c}\right)$ and large separations between bound states in vortices, which will make the operation temperature easier to reach in practice. As topological nontrivial band structures have been predicted to exist in many iron-based superconductors [25-27], $\left(\mathrm{Li}_{1-x} \mathrm{Fe}_{x}\right) \mathrm{OHFeSe}$ with a $T_{c}$ as high as $42 \mathrm{~K}$ appears to be a promising candidate. It belongs to the heavily electrondoped family of iron selenide that only has electron Fermi pockets, and its $E_{F}$ is merely $50-60 \mathrm{meV}$ [28,29]. Unlike $\mathrm{FeTe}_{x} \mathrm{Se}_{1-x}$, the superconducting FeSe layers in $\left(\mathrm{Li}_{1-x} \mathrm{Fe}_{x}\right) \mathrm{OHFeSe}$ are stoichiometric [Fig. 1(a)]. The remaining questions are whether it could exhibit topologically nontrivial behavior and if it hosts MZMs. To address these questions, we conduct density functional theory (DFT) and dynamical mean field theory (DMFT) calculations and ARPES measurements on $\left(\mathrm{Li}_{0.84} \mathrm{Fe}_{0.16}\right) \mathrm{OHFeSe}$, and we find nontrivial band inversion and topological surface states. We study the vortex states of $\left(\mathrm{Li}_{0.84} \mathrm{Fe}_{0.16}\right) \mathrm{OHFeSe}$ by lowtemperature scanning tunneling microscopy (STM). In the core of impurity-free vortices, we indeed discover discrete CdGM states, which are clearly separated from a unique state located exactly at zero bias. Such a clean and robust zero-energy mode exists in all the free vortex cores of $\left(\mathrm{Li}_{0.84} \mathrm{Fe}_{0.16}\right) \mathrm{OHFeSe}$ and matches all expectations of $\mathrm{Fu}$ and Kane's theory, providing an ideal platform to further explore the properties and applications of MZMs.

\section{RESULTS}

\section{A. Electronic structure calculations and ARPES measurements}

In Fig. 1(b), we present our DFT combined with DMFT calculations of the electronic structure of $\left(\mathrm{Li}_{0.75} \mathrm{Fe}_{0.25}\right) \mathrm{OHFeSe}$ (details of the calculation are described in the Appendix and Sec. S1 of Ref. [30]). Along the $\Gamma-Z$ direction, the three flat bands around the Fermi level are dominated by the Fe $3 d_{x y}, 3 d_{x z}$, and $3 d_{y z}$ orbitals, whereas the dispersive band is composed of the Se $4 p_{z}$ orbital and the $3 d_{z}^{2}$ orbital of $\mathrm{Fe}$ in the $\left(\mathrm{Li}_{1-x} \mathrm{Fe}_{x}\right) \mathrm{OH}$ layer. This dispersive $\mathrm{Se} 4 p_{z}$ band has odd parity at the $\Gamma$ and $Z$ points. It crosses the Fermi level and the Fe $3 d$ bands along $\Gamma-Z$, giving rise to a nontrivial band inversion and band topology. Here, Fe atoms in the $\left(\mathrm{Li}_{1-x} \mathrm{Fe}_{x}\right) \mathrm{OH}$ layer play an important role as they hybridize strongly with the Se $4 p_{z}$ orbital and change the dispersion and position of the Se $4 p_{z}$ band. Without the $\mathrm{Fe}$ atom in the $\left(\mathrm{Li}_{1-x} \mathrm{Fe}_{x}\right) \mathrm{OH}$ layer, the band inversion along the $\Gamma-Z$ direction would not exist and the band topology would be trivial [as demonstrated in Fig. S1(b)]. The spin-orbital coupling (SOC) also plays an important role. Without $\mathrm{SOC}$, the $\mathrm{Fe} 3 d_{x z}$ and $3 d_{y z}$ bands are degenerate at the $\Gamma$ point and are in the $\Gamma_{5}^{+}$states, whereas the dispersive $\operatorname{Se~} 4 p_{z}$ band is in the odd $\Gamma_{2}^{-}$state at the $\Gamma$ point. With SOC, the doubly degenerate $\Gamma_{5}^{+}$states are separated into a singlet low-lying $\Gamma_{6}^{+}$state and an upper $\Gamma_{7}^{+}$ state, and the $\Gamma_{2}^{-}$state changes to the $\Gamma_{6}^{-}$state [26]. Under the $\mathrm{C}_{4 v}$ symmetry inherent in the tetragonal crystal structure, the two $\Gamma_{6}^{+}$and $\Gamma_{6}^{-}$state-derived $\Lambda_{6}$ bands with dominating Fe $3 d_{x z}$ and $\mathrm{Se} 4 p_{z}$ orbital characters hybridize with each other along the $\Gamma-Z$ direction and open a gap close to $2.5 \mathrm{meV}$ around their crossing point [marked by the circle in Fig. 1(b)]. The $Z_{2}$ invariant is calculated to be 1 after this SOC gap opens [26], which suggests $\left(\mathrm{Li}_{0.75} \mathrm{Fe}_{0.25}\right) \mathrm{OHFeSe}$ is in a topologically nontrivial phase. Since one cannot make a transition between two states with different topologies without closing the band gap-namely, here between a topological nontrivial bulk with band inversion and the topological trivial "insulating" vacuum-the transition region, i.e., surface, would host gapless topological surface 
(a)

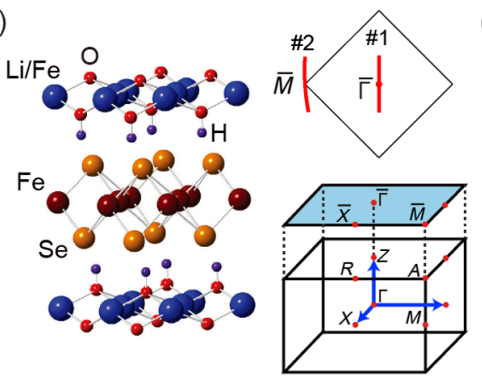

(b)

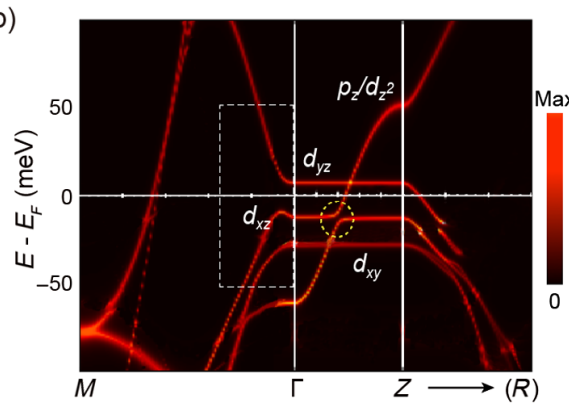

(d) $\quad(\bar{M}) \longleftarrow \bar{\Gamma} \longrightarrow(\bar{M})$

(g) Double-peak fit

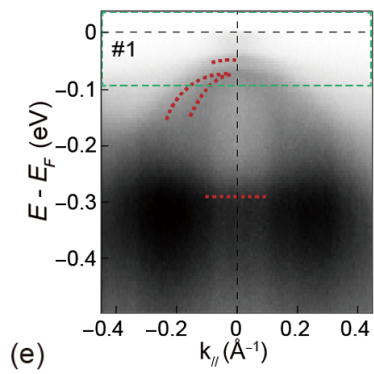

(e)

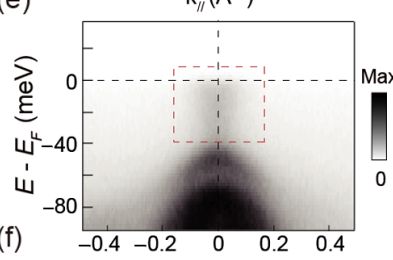

(

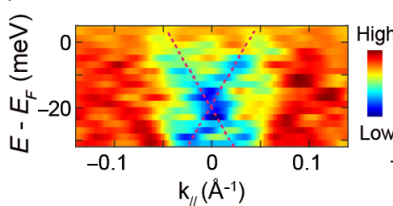

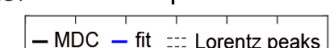

(h)

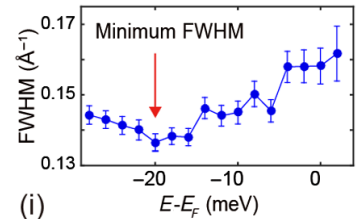

(i)

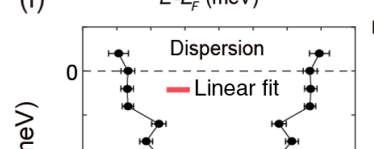

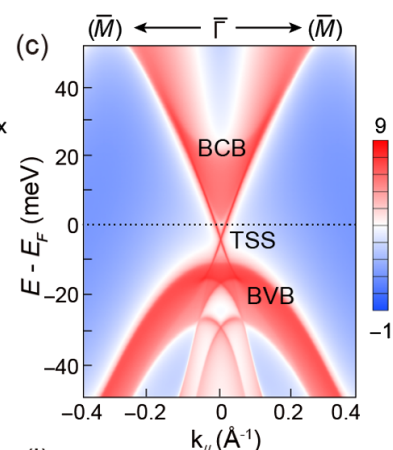

(j)

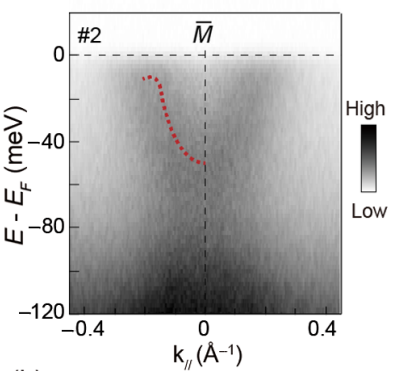

(k)

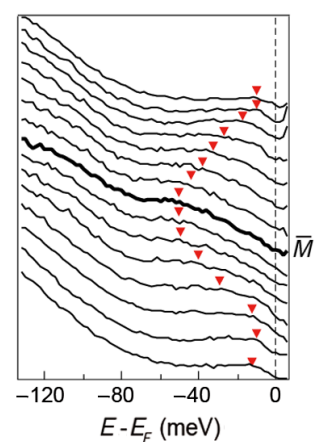

FIG. 1. Band structure and topological surface states of $\left(\mathrm{Li}_{1-x} \mathrm{Fe}_{x}\right) \mathrm{OHFeSe}$. (a) Crystal structure (left), surface Brillouin zone (upper right), and schematic of the projection from the $3 \mathrm{D}$ to $2 \mathrm{D}$ Brillouin zone on the (001) surface (lower right) of $\left(\mathrm{Li}_{1-x} \mathrm{Fe}_{x}\right) \mathrm{OHFeSe}$. (b) Band structure of $\left(\mathrm{Li}_{0.75} \mathrm{Fe}_{0.25}\right) \mathrm{OHFeSe}$ along the $M-\Gamma-Z-(R)$ direction, represented by spectral functions calculated by DFT combined with DMFT methods [more extended band structure is shown in Fig. S1(a)]. The dashed circle indicates the small SOC gap at the crossing point of $\mathrm{Se} 4 p_{z}$ and $\mathrm{Fe} 3 d_{x z}$ bands. The dashed rectangle corresponds to the left half of the energy-momentum range of panel (c). (c) Calculated bulk and surface states on the (001) surface along the $\bar{\Gamma}-\bar{M}$ direction, illustrated by the spectral function. Dirac-cone-like topological surface states (TSS) centered at $\bar{\Gamma}$ connect the bulk valence band (BVB) and conduction band (BCB). The $\mathrm{BVB}$ and $\mathrm{BCB}$ are mainly composed of $3 d_{x z}$ and $3 d_{y z}$ orbitals along this direction, respectively. (d) Photoemission intensity of $\left(\mathrm{Li}_{0.84} \mathrm{Fe}_{0.16}\right)$ OHFeSe measured across $\bar{\Gamma}$ along cut \#1 in panel (a). The dashed curves show the bulk band structure determined by second derivatives. The green dashed rectangle corresponds to the energy-momentum range of panel (e). (e) An enlargement of data in panel (d) near $E_{F}$ at the $\bar{\Gamma}$ point. Finite spectral weight can be clearly observed within the bulk band gap. (f) Second derivative of the photoemission intensity in the marked region of panel (e), where a Dirac-cone-like dispersion can be seen. (g) Momentum distribution curves (MDCs) of the data in panel (e) in the energy range of $-28 \mathrm{meV}-2 \mathrm{meV}$ (normalized by the intensity near $\mathrm{k}_{/ /}=0$ ). Red markers indicate the Dirac-cone-like dispersion extracted from the two-Lorentzian-peak fitting (blue and dashed curves). (h) The FWHM obtained from the one-peak fit [shown in Fig. S2(a)], as a function of energy. Error bars represent 95\% confidence bounds. (i) $E-k$ dispersion extracted from the two-peak fit in panel (g), and the linear fit around the crossing point. Error bars represent $95 \%$ confidence bounds of the fitted peak position. (j) Photoemission intensity taken along cut \#2 across $\bar{M}$ in panel (a). The dashed curve shows the dispersion of the bulk electron band. (k) The energy distribution curves (EDCs) of the data in panel (j) after dividing by Fermi-Dirac distribution. (1) Symmetrized EDC near the Fermi crossing of the $M$ pocket, where a superconducting gap of around $10 \mathrm{meV}$ is observed (indicated by red arrows). All the data were measured at $5.6 \mathrm{~K}$ using $21.2-\mathrm{eV}$ photons.

states (TSS) in the band gap. More specifically, the hybridization gap between the $\Gamma_{6}^{+}$and $\Gamma_{6}^{-}$states at the surface is removed by the discontinuity of the surface. Indeed, our calculations show that Dirac-cone-like surface states centered at $\bar{\Gamma}$ appear on the (001) surface as shown in Fig. 1(c). The topological surface states have helical spin texture and can produce the MZM when it is in close proximity to $s$-wave superconductivity $[15,26,27]$. 
Guided by these calculations, we have conducted further ARPES measurements on high-quality $\left(\mathrm{Li}_{0.84} \mathrm{Fe}_{0.16}\right) \mathrm{OHFeSe}$ films grown on $\mathrm{LaAlO}_{3}$ by hydrothermal epitaxy $\left(T_{c} \sim 42 \mathrm{~K}\right)$ [31,32] (see the Appendix for details). Figure 1(d) shows the ARPES spectrum of a cleaved film across the Brillouin zone (BZ) center as shown in Fig. 1(a). Multiple parabolic bands can be clearly resolved below the Fermi surface, and there is one flat band around $-300 \mathrm{meV}$. Moreover, an electron band with its band bottom at about $-50 \mathrm{meV}$ is observed at $M$ [Figs. 1(j) and 1(k)]. These observed bands and the ones calculated in Fig. 1(b) have one-to-one correspondence, although qualitatively. The relative positions and bandwidths may differ due to correlation effects. We also note that certain bands, such as the other electronlike band at $M$, were not observed here due to known matrix element effects. A precise band calculation for heavily electron-doped iron selenides is still challenging; however, qualitative features of the measured bands are reproduced by the calculation in Fig. 1(b), and the generic features here resemble several iron chalcogenide superconductors [33].

Intriguingly, in the region near the Fermi energy as plotted in Fig. 1(e), some finite spectral weight within the bulk band gap can be clearly observed. The second derivative of the region containing this spectral weight [Fig. 1(f)] displays an X-shaped band structure. Figure 1(g) shows the momentum distribution curves (MDCs) of the data in Fig. 1(e) near $E_{F}$. Through a one-Lorentzianpeak fit [shown in Fig. S2(a)], we found that the width of the spectral peak near $k_{/ /}=0$ has a minimum at $E=$ $-20 \mathrm{meV}$ [Fig. 1(h)]. This would indicate the position of the band crossing point. Then, we apply a two-peak fit to MDCs, assuming each peak has a constant FWHM like that of the single peak at $E=-20 \mathrm{meV}$ (the scattering rate variations are expected to be negligible in such a small energy window). The results display a nearly linear, Diraccone-like band dispersion, as shown in Fig. 1(g), and its overall fitting quality is better than that of the one-peak fit [see Fig. S2(c) for comparison]. In Fig. 1(i), we plot the $E-k$ relations extracted from the two-peak fit; a linear fit is found to be plausible near the crossing point, which gives $v_{F}=5.5( \pm 1.5) \times 10^{4} \mathrm{~m} / \mathrm{s}$ and $E_{D}=20( \pm 2) \mathrm{meV}$ [see Fig. S2(d) for more details]. The $k_{F}$ is determined to be about $0.028 \AA^{-1}$ through the MDC near $E_{F}$.

We note that the existence of topological surface states is determined by the band topology. The qualitative agreement between the measured and calculated band structures throughout BZ would suggest that they have the same topology. Therefore, the predicted topological surface states provide the most natural explanation for such a nearly linear, Dirac-cone-like band dispersion at $\Gamma$. Nevertheless, one should examine whether it is related to any other known electron pockets in the $\left(\mathrm{Li}_{1-x} \mathrm{Fe}_{x}\right) \mathrm{OH}$ and FeSe surface layers exposed by cleavage. On the FeSe surface layer, an electronlike pocket would only appear around $\Gamma$ after heavily doping electrons through potassium dosing, and thus it is well above the Fermi energy in the case considered here [34]. There is possibly an electron pocket around $\Gamma$ in the $\left(\mathrm{Li}_{1-x} \mathrm{Fe}_{x}\right) \mathrm{OH}$ surface layer, based on our previous quasiparticle interference (QPI) measurements of $\left(\mathrm{Li}_{0.8} \mathrm{Fe}_{0.2}\right)$ OHFeSe bulk crystal [35]. However, its band bottom is at about $50 \mathrm{meV}$ below $E_{F}$ with a much larger Fermi velocity, so it cannot be the Dirac cone observed here. Therefore, we conclude that the observed Dirac band dispersion can only be attributed to the topological surface state predicted in our calculations.

The FeSe surface of $\left(\mathrm{Li}_{1-x} \mathrm{Fe}_{x}\right) \mathrm{OHFeSe}$ is fully gapped [35,36], as shown in Fig. 2 below. If this topological surface state is on the FeSe surface, it should be gapped. A superconducting gap of about $10 \mathrm{meV}$ can be observed on the electronlike band around $\bar{M}$, as shown in Figs. 1(j) and 1(k), and further illustrated in Fig. 1(l) (details are described in the caption). The absence of a gap in our data for the topological surface state around $\bar{\Gamma}$ may be attributed to the fact that this gap is small, while the resolution of ARPES is about $6 \mathrm{meV}$. Moreover, ARPES collects data over a millimeter-sized region and thus contains a large contribution from the nonsuperconducting and disordered $\left(\mathrm{Li}_{1-x} \mathrm{Fe}_{x}\right) \mathrm{OH}$ surface, which has low-energy spectral weight near $\bar{\Gamma}$.

\section{B. STM characterization of the sample surface and superconducting gap}

STM measurement was conducted in a cryogenic STM with a base temperature of $0.4 \mathrm{~K}$ and had an energy resolution of $0.36 \mathrm{meV}$ (see the Appendix and Ref. [30], Sec. S3). Figure 2(a) shows typical topography of a cleaved $\left(\mathrm{Li}_{0.84} \mathrm{Fe}_{0.16}\right) \mathrm{OHFeSe}$ film. We observed both FeSe-terminated (left) and $\left(\mathrm{Li}_{1-x} \mathrm{Fe}_{x}\right) \mathrm{OH}$-terminated (right) surfaces. The inset of Fig. 2(a) shows the atomically resolved FeSe lattice with $a_{0}=3.8 \AA$. Dimerlike defects at the Fe site were frequently observed (dashed circle) and are likely substitutional impurities. The typical tunneling spectrum $(d I / d V)$ of the FeSe surface is shown in Fig. 2(b) (blue curve). It displays a full superconducting gap with two pairs of coherence peaks (referred to as $\Delta_{1}$ and $\Delta_{2}$ ) and a flat bottom, as observed in bulk crystals [35,36]. Since our band calculations and ARPES measurements reveal surface states at $\bar{\Gamma}$, it is possible that the double gaps actually originate from the bulk and the surface band, as discussed below. For the $\left(\mathrm{Li}_{1-x} \mathrm{Fe}_{x}\right) \mathrm{OH}$ surface, the tunneling spectrum typically shows metallic behavior with a weak dip off the Fermi level [green curve in Fig. 2(b)].

To determine the gap of the FeSe surface more precisely, we fit the tunneling spectrum with a Dynes function (see Sec. S4 of Ref. [30] for details). The observed gaps are broader than an isotropic gap with a similar size [grey curve in Fig. 2(c)]. However, the line shape of the smaller gap $\left(\Delta_{1}\right)$ can be well fitted by an empirical anisotropic gap function: $\Delta_{1}(\mathrm{k})=\Delta_{1}^{\min }+\left(\Delta_{1}^{\max }-\Delta_{1}^{\min }\right)|\cos 2 \theta|$ [red curve 
(a)

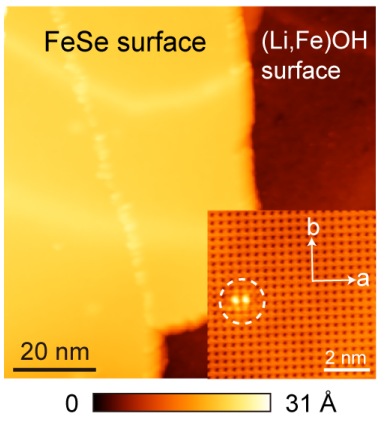

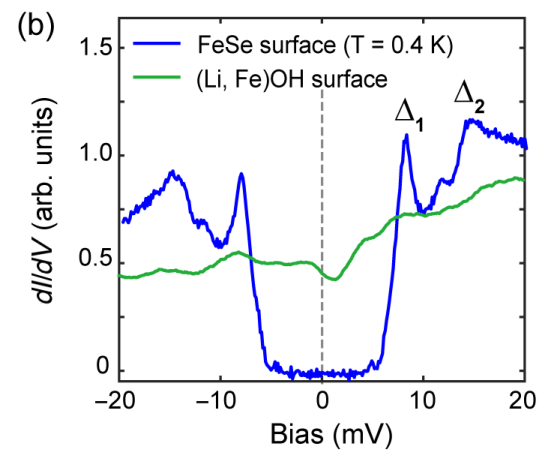

(c)

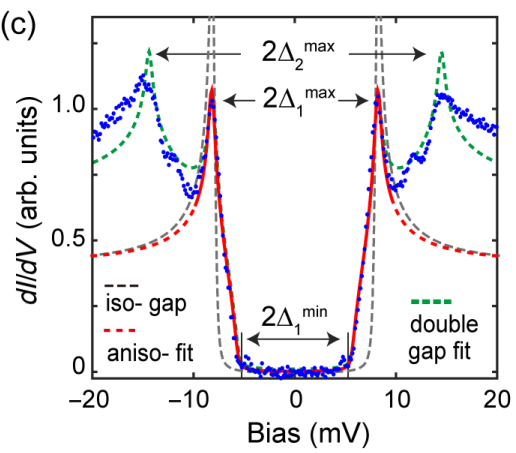

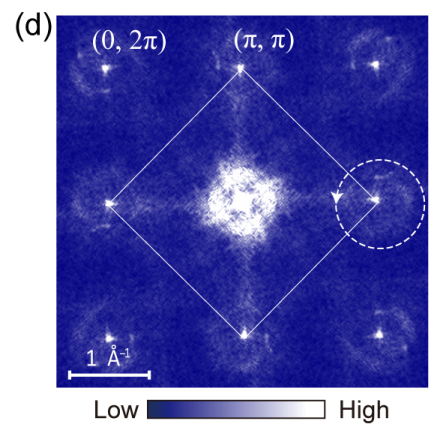
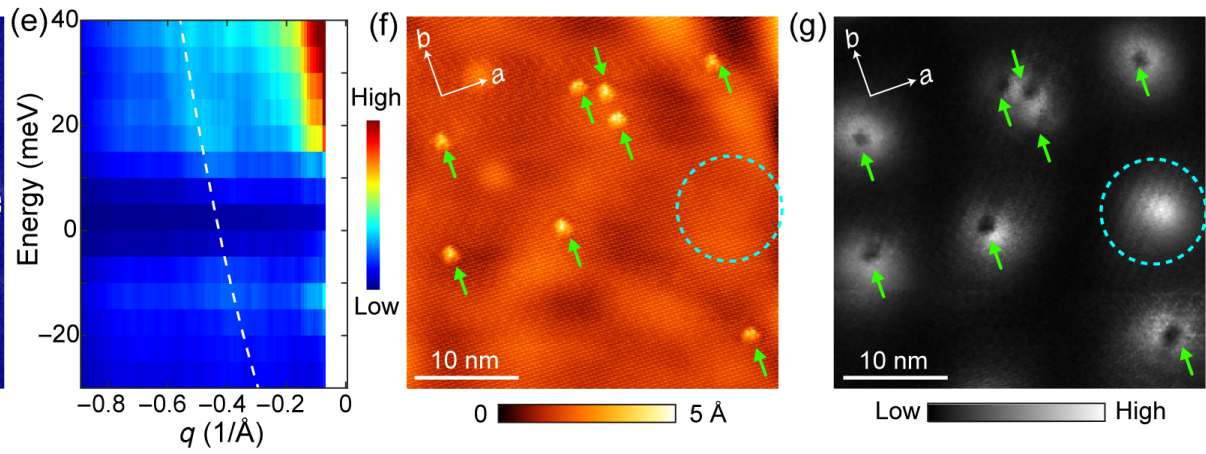

FIG. 2. Topography, superconducting gap, QPI, and vortex mapping of $\left(\mathrm{Li}_{0.84} \mathrm{Fe}_{0.16}\right) \mathrm{OHFeSe}$ film. (a) Topographic image of a cleaved film $\left(V_{b}=1 \mathrm{~V}, I=5 \mathrm{pA}\right)$. Inset: The lattice of the FeSe surface $\left(V_{b}=50 \mathrm{mV}, I=20 \mathrm{pA}\right)$. A dimerlike defect is marked by the circle. (b) Typical $d I / d V$ spectra taken on the FeSe surface at $0.4 \mathrm{~K}\left(V_{b}=-20 \mathrm{mV}, I=60 \mathrm{pA}\right)$ and on the $\left(\mathrm{Li}_{0.84} \mathrm{Fe}_{0.16}\right) \mathrm{OH}$ surface at $4.2 \mathrm{~K}$ $\left(V_{b}=-20 \mathrm{mV}, I=60 \mathrm{pA}\right)$. Note that $\Delta_{1}$ and $\Delta_{2}$ refer to the smaller and larger gaps, respectively. (c) Fits of the superconducting gap (blue dots) to a single isotropic gap function (gray dashed curve), a single anisotropic gap function (red curve), and a double anisotropic gap function (green dashed curve). Details are described in Ref. [30]. (d) Symmetrized FFT-QPI pattern of an FeSe surface taken at $V_{b}=5 \mathrm{mV}$ (mapping size: $36 \times 36 \mathrm{~nm}^{2}$ ). (e) Color plot of FFT line cuts through $q=(\pi, \pi)$ [azimuth averaged along the dashed circle in panel (d)]. The dashed curve is a parabolic fit to the electronlike dispersion. (f) Topographic image of an FeSe surface $\left(V_{b}=-30 \mathrm{mV}, I=40 \mathrm{pA}\right.$; size: $\left.36 \times 36 \mathrm{~nm}^{2}\right)$; green arrows highlight dimerlike defects. $(\mathrm{g}) \mathrm{ZBC}$ map of the area in panel (f), under $B=10 \mathrm{~T}$. Pinned vortices are indicated by arrows. The dashed circle encloses a free vortex. If we assume that each vortex carries a flux quantum $\Phi_{0}=\mathrm{h} / 2 \mathrm{e}$, this area should contain $(\mathrm{B} \times \mathrm{S}) / \Phi_{0}=6.3$ vortices, consistent with the observed number of 7 .

in Fig. 2(c)] after subtracting a sloping background. The fit yields $\Delta_{1}^{\max }=8.2 \mathrm{meV}, \Delta_{1}^{\min }=5.7 \mathrm{meV}$, and a small Dynes term $\Gamma$ of $0.1 \mathrm{meV}$. Note that such a $\Delta_{1}^{\max }$ value can also be estimated as half the distance between the two corresponding coherence peaks, as can $\Delta_{2}^{\max }$. However, the larger gap $\Delta_{2}$ has additional broadening, which makes the determination of gap anisotropy difficult. The green curve in Fig. 2(c) gives a simulated spectrum with $\Delta_{2}^{\max }=$ $14.5 \mathrm{meV}$ (see Ref. [30] for details). We note that the size of $\Delta_{1}$ (even $\Delta_{1}^{\max }$ ) is obviously smaller than the ARPES measured gap $(\sim 10 \mathrm{meV})$ on the $\bar{M}$ pocket; thus, $\Delta_{1}$ is unlikely from the $\bar{M}$ pocket, and it can only be attributed to the gap of the topological surface state obtained through proximity from the bulk. On the other hand, $\Delta_{2}^{\max }$ is larger than $10 \mathrm{meV}$, and thus $\Delta_{2}$ should correspond to the bulk gap on the $\bar{M}$ pocket. As will be shown below, the gap size measured by ARPES reflects the average size of $\Delta_{2}$.

QPI measurements were carried out on the FeSe surface. Figure 2(d) presents a typical fast-Fourier-transformed (FFT) QPI image taken at $E=5 \mathrm{meV}$ and $T=4.2 \mathrm{~K}$-more data are presented in Sec. S5 of Ref. [30]. The dominant features are the ringlike patterns centered at $(0,0),(\pi, \pi)$, and $(0,2 \pi)$, which arise from the scattering between electron pockets at $\bar{M}[35,36]$. Figure 2(e) summarizes the averaged FFT line cuts through $q=(\pi, \pi)$, which displays an electronlike dispersion. Using $q=2 k$, a parabolic fit yields a band bottom at $-57( \pm 7) \mathrm{meV}$ and $k_{F}=0.21( \pm 0.02) \AA^{-1}$, consistent with the reported band structure at $\bar{M}$ of $\left(\mathrm{Li}_{1-x} \mathrm{Fe}_{x}\right)$ OHFeSe [28,29]. In relation to the topological surface states at $\bar{\Gamma}$, we note that a spin-helical structure with spin-momentum locking would strongly suppress backscattering, causing them to be invisible to QPI.

Under a magnetic field of $10 \mathrm{~T}$ perpendicular to the surface, a zero-bias-conductance (ZBC) map is taken on a $36 \times 36 \mathrm{~nm}^{2}$ area of FeSe surface [Fig. 2(f)], as shown in Fig. 2(g). Vortex cores are visible as bright circular regions. We find that many of the vortices are pinned by dimerlike defects, as indicated by the corresponding arrows in Figs. 2(f) and $2(\mathrm{~g})$. The pinned vortices all have "dark-spot"-like features near their centers, which are induced by impurity states. However, we can still find unpinned or "free" vortices 

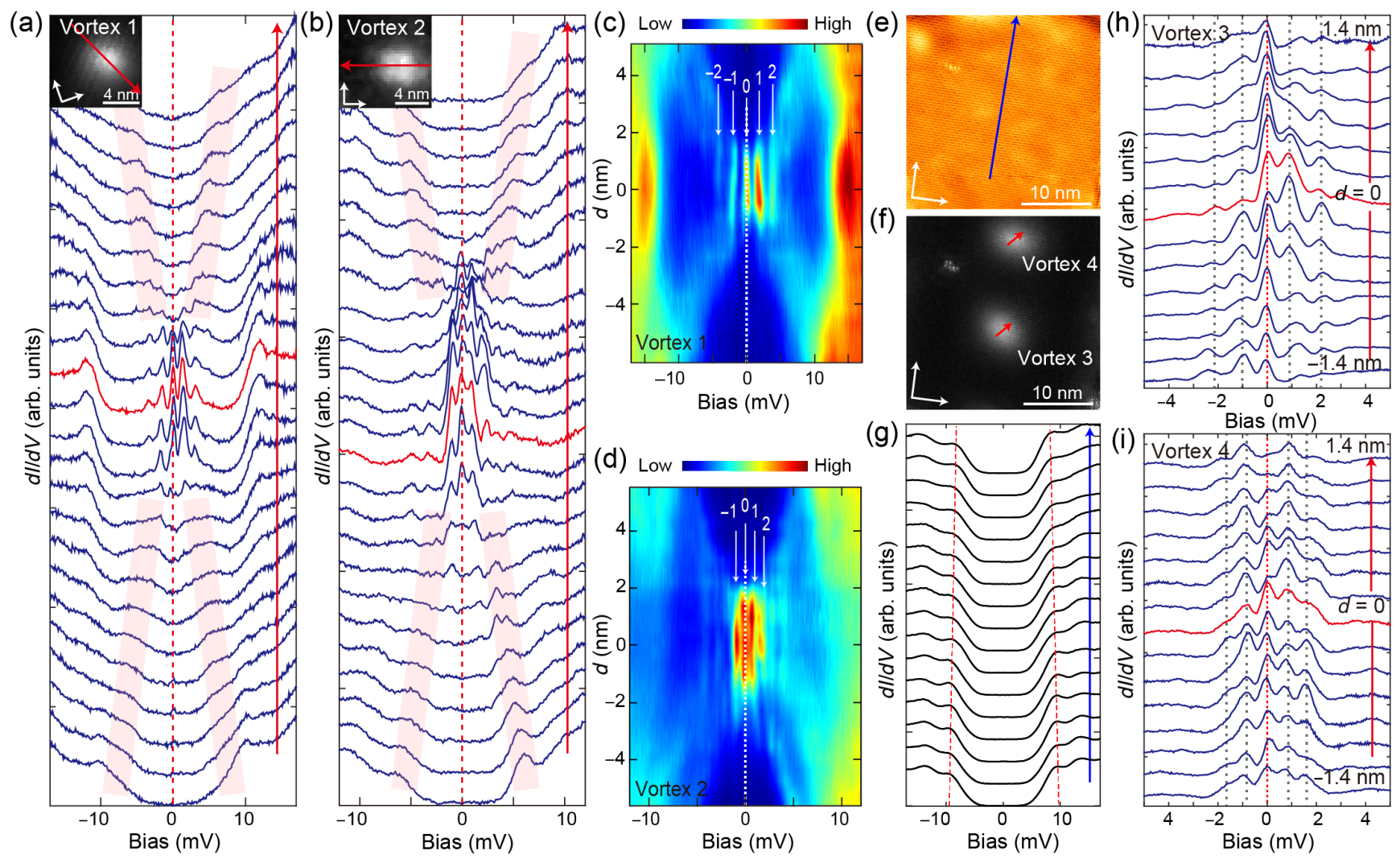

FIG. 3. Bound states in the free vortex cores. (a,b) $d I / d V$ spectra taken across the free vortices 1 and 2 , shown in the inset images, respectively (set point: $V_{b}=-17 \mathrm{mV}, I=60 \mathrm{pA}$ ). (c,d) Color plots of the spatial dependence of the $d I / d V$ spectra shown in panels (a) and (b), respectively. Dashed lines indicate the zero bias, and arrows indicate the positions of discrete low-energy states. (e,f) Topographic image and vortex mapping $(B=10 \mathrm{~T})$ of an FeSe surface, respectively. Two free vortices $(3$ and 4$)$ are observed in this region. (g) $d I / d V$ spectra taken along the blue arrow in panel (e), at $T=4.2 \mathrm{~K}$ and $B=0 \mathrm{~T}$. Red dashed lines trace the position of inner coherence peaks. (h,i) $d I / d V$ spectra taken along the red arrows in panel (f) across the center of vortices 3 and 4 , respectively (set point: $\left.V_{b}=-5 \mathrm{mV}, I=60 \mathrm{pA}\right)$. Dashed lines indicate peak positions at the vortex center.

that emerge in defect-free areas, such as the one marked by a dashed circle in Fig. 2(g).

\section{Discrete bound states in the free (unpinned) vortex cores}

The tunneling data of four different free vortices are presented in Fig. 3. Figure 3(a) plots the $d I / d V$ line cut taken across vortex 1 (inset image, along the arrow); the red curve is measured at the vortex center. It is remarkable that multiple (five) discrete peaks are observed near the core center. There is one peak located exactly at zero bias (a ZBCP), with the other peaks distributed symmetrically around it. The energy spacing between these 5 peaks is close to $1.5 \mathrm{meV}$. Figure 3(c) presents the spatial evolution of the spectra in a color plot. On leaving the core center, the intensities of these discrete peaks (marked by $-2,-1,0,1$, 2 here, also referred to as $E_{-2}, E_{-1}, E_{0}, E_{1}, E_{2}$ hereafter) decrease and vanish at about $2 \mathrm{~nm}$ away. Intriguingly, when the discrete peaks fade out, a pair of much broader peaks [shaded regions in Fig. 3(a)] show up at higher energies. Those peaks keep shifting to higher energies on moving away from the core, giving an X-shaped pattern in Fig. 3(c). Figures 3(b) and 3(d) present similar data taken on another free vortex (vortex 2). Multiple low-energy core states including a ZBCP were also observed, albeit with a slightly smaller energy spacing $(1 \mathrm{meV})$, and there are also "shifting" high-energy states away from the core center. The behavior of these states, except the one at zero bias, are expected for the CdGM vortex states in the quantum limit $[13,37]$.

Such a ZBCP accompanied by discrete CdGM states was repeatedly observed in free vortices. In regions with few defects, such as the one shown in Fig. 3(e), more than one free vortex (vortices 3 and 4) could be found [Fig. 3(f)]. At zero field, a clean superconducting gap is observed over this region, which could vary by about $2 \mathrm{meV}$ along the long cut [Fig. 3(g)]. Figures 3(h) and 3(i) show the $d I / d V$ line cuts of vortices 3 and 4, respectively, focusing on the region close to the vortex center $( \pm 1.4 \mathrm{~nm})$ and low energies $( \pm 5 \mathrm{meV})$. There are five discrete peaks with a ZBCP in most spectra, with the energy spacing of $0.8-1.0 \mathrm{meV}$, well above our energy resolution $(0.36 \mathrm{meV})$. 

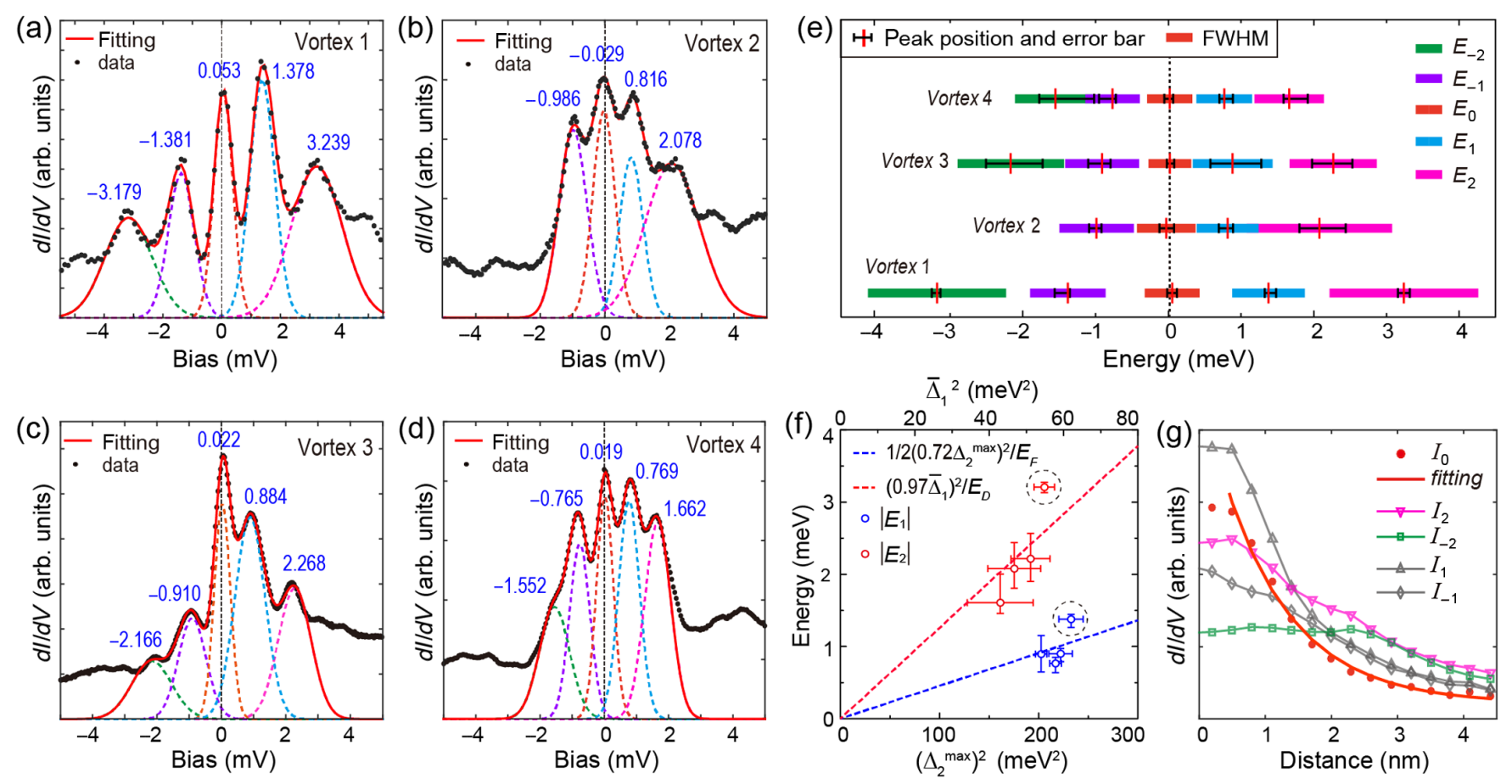

FIG. 4. Quantitative characterization of the vortex core states. (a)-(d) Summed low-energy $d I / d V$ spectra taken near the centers of vortices 1-4. Black dots are experimental data. Red solid curves are the fits to multiple Gaussian peaks (dashed curves are the individual peaks). (e) Peak energy position (marked by red vertical rods) and FWHM (represented by the length of colored bars) of the core states of vortices 1-4, obtained from Gaussian peak fitting. The black error bars represent the ranges of peak energy fluctuations (see Sec. S8 of Ref. [30] for more details). (f) Plots of $\left|E_{2}\right|$ (red circles) as a function of $\left(\overline{\Delta_{1}}\right)^{2}$ and $\left|E_{1}\right|$ (blue circles) as a function of $\left(\Delta_{2}^{\max }\right)^{2}$. Dashed lines are the linear fitting (see legend). (g) Spatial dependence of the intensity of the core states. Here, $I_{2}, I_{1}, I_{0}, I_{-1}$, and $I_{-2}$ represent the intensity $\left(d I / d V\right.$ values at the peak energy) of the five core states $E_{2}, E_{1}, E_{0}, E_{-1}$, and $E_{-2}$ of vortex 1 . The red solid curve is the exponential fit to the decay of $E_{0}$.

The positions of $E_{-2}, E_{-1}, E_{1}$, and $E_{2}$ peaks vary in different vortices, and they even vary at different locations in the same vortex. As shown in Figs. 3(h) and 3(i), upon leaving the center, the positions of $E_{-2}, E_{-1}, E_{1}$, and $E_{2}$ peaks shift away from the dashed lines that represent the peak positions at the center. This is because the CdGM states are spaced by $\delta E=\Delta^{2} / E_{F}$, and the superconducting gap varies in space [Fig. 3(g)]. In contrast, the energy position of the $E_{0}$ peak is always at zero bias, independent of the local gap, which suggests that it is most likely protected by some global properties, such as topology. In addition, since conventional CdGM states are not located at zero energy, but at half-integer multiples of $\Delta^{2} / E_{F}$, the origin of the $E_{0}$ peak is highly nontrivial.

The high-level core states are closely packed and form the broad "shifting" peaks. In theory, the spacing between high-level states will decrease and make them undistinguishable, while the maximum intensity of core states will shift to high energy on leaving the core center [37-39], resulting in two "splitting" peaks. This behavior has been widely observed, such as in $\mathrm{NbSe}_{2}$ (Ref. [38]); however, individual core states are rarely seen due to the small $\delta E=$ $\Delta^{2} / E_{F}$ for most conventional superconductors. Here, we clearly observe both discrete low-level core states and the quasicontinuous high-level states, benefitting from the relatively large $\delta E$ of $\left(\mathrm{Li}_{1-x} \mathrm{Fe}_{x}\right) \mathrm{OHFeSe}$ (as shown below) and sufficiently high resolution at $0.4 \mathrm{~K}$ (for comparison, see Ref. [30], Sec. S6, for the $\mathrm{T}=4.2 \mathrm{~K}$ data).

The well-separated low-level core states enable quantitative analysis. We first apply multiple-Gaussian-peak fitting to the summed low-energy spectra taken near the vortex center (over a range of about $\pm 0.7 \mathrm{~nm}$ to reduce the uncertainty from peak fluctuations). The results are shown in Figs. 4(a)-4(d) for vortices 1-4, respectively. Here, each core-state peak corresponds to one Gaussian peak. The fitted peak energies are directly labeled in Figs. 4(a)-4(d) and are also illustrated in Fig. 4(e) with error bars that represent the energy range of peak fluctuation. The full widths at half maximum (FWHM) of the Gaussian peaks are illustrated in Fig. 4(e) by colored bars. The detailed values of all the fit parameters are listed in Table SI in Ref. [30]. From the fitting, we can reveal several important facts.

First, the fitted energy of $E_{0}$ for all the vortices is negligibly small, typically 1 order of magnitude smaller than the energy resolution $(0.36 \mathrm{meV})$. The fluctuation range of $E_{0}$ [error bars in Fig. 4(e)] always covers zero. Meanwhile, the FWHM of the $E_{0}$ peak is in the range of 
$0.59-0.80 \mathrm{meV}$, larger than the energy resolution. However, one cannot attribute such an additional broadening (0.23-0.44 meV) to two unresolved conventional CdGM states sitting close to zero bias, assuming they have the energy of $\pm \frac{1}{2} \Delta^{2} / E_{F}$, because it would require a superconducting gap with a mean size of 3.6-5.0 meV (assuming $E_{F}=57 \mathrm{meV}$ ), and the observed local gap size near these vortices is well above that value [see Fig. S6 and Table SII; the size of $\overline{\Delta_{1}}=\left(\Delta_{1}^{\max }+\Delta_{1}^{\min }\right) / 2$ is around $\left.7 \mathrm{meV}\right]$. In addition, the FWHM of $E_{0}$ is obviously narrower than the FWHM of other CdGM states. As discussed below, the gap anisotropy may induce additional broadening but cannot account well for the finite width of $E_{0}$. Therefore, within our experimental resolution, $E_{0}$ is a zero-energy state with finite width, whose origin is yet to be explored.

Second, the energies of the $E_{ \pm 2}$ and $E_{ \pm 1}$ states are nearly symmetric with respect to the Fermi level (with different values for different vortices). However, the spacing between $E_{2}$ and $E_{1}$ is always slightly larger than that between $E_{1}$ and $E_{0}$. The ratio of $\left(E_{2}-E_{-2}\right) /\left(E_{1}-E_{-1}\right)$ falls between 2.1 and 2.5 for vortices $1-4$ (see Table SII). This is unexpected if we assume all five states are from one single electronic component since the spacing between neighboring states will decrease when going to higher energy [37,39]. Therefore, these may actually arise from two bands with different gap size and/or band bottom position, as we observe a double gap $d I / d V$ spectrum and a topological surface state. Meanwhile, the local gap inhomogeneity and anisotropy should also affect the energy of the core state for different vortices. Taking into account these effects, we measure the local gap size $\left(\Delta_{1}^{\max }, \Delta_{1}^{\min }\right.$, and $\Delta_{2}^{\max }$ ) of the region where these vortices emerge (see Fig. S6 and Table SII for details). As $\Delta_{1}$ is attributed to the topological surface state, and its approximate mean size is $\overline{\Delta_{1}}=\left(\Delta_{1}^{\max }+\Delta_{1}^{\min }\right) / 2$, considering the Dirac point is at $20 \mathrm{meV}$ below the Fermi level (defined as $E_{D}$ ), then it could reasonably account for the $E_{0}$ and $E_{ \pm 2}$ states for most free vortices through the general formula $E=\mu \Delta^{2} / E_{D}$ and $\mu=0, \pm 1$. In Fig. 4(f), we plot $\left|E_{2}\right|=\left(E_{2}-E_{-2}\right) / 2$ vs $\left(\overline{\Delta_{1}}\right)^{2}$ for different vortices (red spots); a linear fit gives $\left|E_{2}\right|=\left(0.97 \overline{\Delta_{1}}\right)^{2} / E_{D}$. We note that there is a more specific calculation on the vortex states of proximity-induced superconductivity in the topological surface state [40], which gives $E= \pm 0.83 \Delta^{2} /\left(\Delta^{2}+E_{D}^{2}\right)^{1 / 2}$ for the two second-lowest states. This formula can estimate $E_{ \pm 2}$ states by taking $\Delta \sim 1.06 \overline{\Delta_{1}}$, which is also in between $\Delta_{1}^{\min }$ and $\Delta_{1}^{\max }$ (see Table SII).

For the topologically trivial bulk state around $\mathrm{M}$ with $E_{F}=57 \mathrm{meV}$, its gap is $\Delta_{2}$. Its lowest-order core states should be at $\pm \frac{1}{2} \Delta_{2}^{2} / E_{F}$. The mean value of $\Delta_{2}$ is difficult to determine, but $\Delta_{2}^{\max }$ can be estimated by the coherence peaks. We find that a linear fit of $\left|E_{1}\right|=\left(E_{1}-E_{-1}\right) / 2$ vs $\left(\Delta_{2}^{\max }\right)^{2}$ yields $\left|E_{1}\right|=\frac{1}{2}\left(0.72 \Delta_{2}^{\max }\right)^{2} / E_{F}$ [blue dashed line in Fig. 4(f)]. The value of $0.72 \Delta_{2}^{\max } \approx 10 \mathrm{meV}$ is consistent with the ARPES gap size on the band around the $M$ point [Figs. 1(j) and 1(k)], which should be close to $\overline{\Delta_{2}}$. Although the two data points marked by dashed circles deviate from the fits, they still show a monotonic relation with the gap. We speculate that this could be due to some local variation of carrier concentration, which is difficult to determine precisely for each vortex. Despite this uncertainty, the most self-consistent understanding is that the $E_{0}$ and $E_{ \pm 2}$ are from the topological surface state, while $E_{ \pm 1}$ are from the trivial bulk band.

Third, the fitted FWHMs of the $E_{ \pm 2}$ and $E_{ \pm 1}$ states vary between 0.8 and $2 \mathrm{meV}$. We expect that this is at least partially due to the sizable anisotropy of $\Delta_{1}$ and $\Delta_{2}$. The broadening of $E_{ \pm 2}$ caused by gap anisotropy of $\Delta_{1}$ [defined by $\left.\alpha=\left(\Delta_{1}^{\max }-\Delta_{1}^{\min }\right) /\left(\Delta_{1}^{\max }+\Delta_{1}^{\min }\right)\right]$ can be estimated by $\delta\left(\Delta_{1}^{2} / E_{\mathrm{D}}\right)=2 \alpha\left(\overline{\Delta_{1}}\right)^{2} / E_{D}$, yielding an additional broadening in the range of $0.7-1.5 \mathrm{meV}$ for vortices $1-4$. Meanwhile, although the gap anisotropy of $\Delta_{2}$ is unknown, if we take the same value with $\alpha$ and assume $\overline{\Delta_{2}}=0.72 \Delta_{2}^{\max }$, its contribution to the FWHM of $E_{ \pm 1}$ is in the range of $0.3-0.6 \mathrm{meV}$. Then, the resulting total broadenings are compatible with the fitted FWHMs. However, the estimated total broadening of $E_{ \pm 2}(1.1-1.9 \mathrm{meV})$ is significantly larger than the FWHM of $E_{0}$, indicative of the special properties of the latter. It is also worth noting that the core states have different but comparable weight, as reflected by the peak area summarized in Table SI. Moreover, the intensities of $E_{ \pm 2}$ and $E_{ \pm 1}$ are asymmetric. This is consistent with the particle-hole asymmetry expected for a superconductor with small $E_{F}[37,41,42]$.

Figure 4(g) shows the spatial dependence of the intensity of the core states $[d I / d V$ values at the peak energies, extracted from Fig. 3(c) for vortex 1]. The $E_{0}$ state is more concentrated at the vortex center (especially compared to $E_{ \pm 2}$ ), consistent with the calculated behavior of the vortex state of a $p+i p$ superconductor $[43,44]$. An exponential fit (red solid curve) gives a decay length of $1.4 \mathrm{~nm}$, which is an estimation of coherence length. Theoretically, the lowlevel core states should have an additional oscillation with a period of $\lambda_{F}=2 \pi / k_{F}$ (characterized by Bessel functions $[43,44])$ besides the exponential decay. Here, $k_{F}$ is $0.21 \AA^{-1}$ for the bulk band and about $0.03 \AA^{-1}$ for the surface band (from QPI and ARPES measurements), which gives $\lambda_{F}^{B}=3.0 \mathrm{~nm}$ and $\lambda_{F}^{S}=21.0 \mathrm{~nm}$. These values are both significantly larger than $\xi$; thus, the $\lambda_{F}$ oscillation cannot be observed here.

\section{Core states of impurity-pinned vortices}

A large number of vortices are pinned by dimerlike defects [Figs. 2(f)-2(g)], as the dimerlike defects at the Fe site can locally suppress superconductivity by inducing impurity states. Figures 5(a) and 5(b) show the tunneling spectra measured at $T=0.4 \mathrm{~K}$ on two typical dimerlike defects under $B=0 \mathrm{~T}$ and $10 \mathrm{~T}$ (or $11 \mathrm{~T}$ ). Multiple, sharp, 

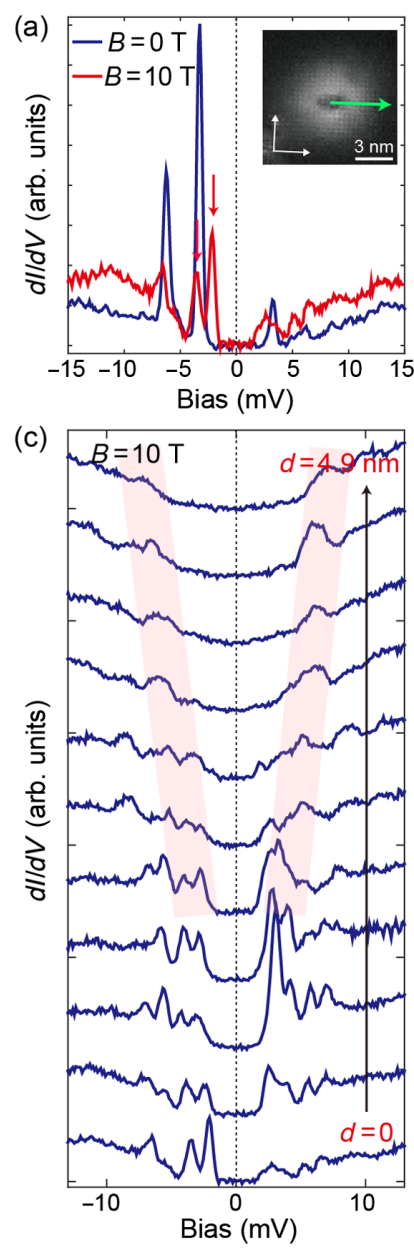

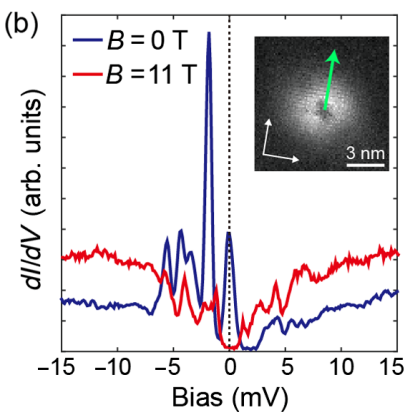

(d)

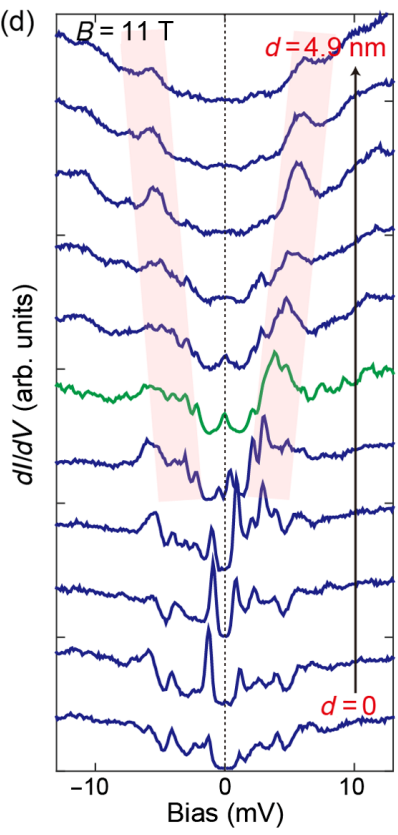

FIG. 5. Bound states in the impurity pinned vortex cores. (a,b) $d I / d V$ spectra measured on two dimerlike defects at $B=0 \mathrm{~T}$ and $B=10(11) \mathrm{T}\left(V_{b}=-17 \mathrm{mV}, I=60 \mathrm{pA}\right)$. Insets in panels (a) and (b): ZBC maps of the pinned vortex. (c,d) $d I / d V$ spectra measured along the arrows in the inset image, respectively.

impurity-state peaks can be observed at $B=0 \mathrm{~T}$ (blue curves), and the peak positions can vary for different defects (see Fig. S8 for additional data). In some cases, a zero-bias peak can show up at the defect site in zero field [Fig. 5(b)]. At a high field, vortices pinned by these two defects are observed in the ZBC map [the insets of Figs. 5(a) and 5(b)]. The spectra on the defect sites now display signatures of split impurity states, such as the double peaks marked by arrows in Fig. 5(a) (see also Fig. S8). The zero-bias peak in Fig. 5(b) is also split away from zero bias. This result suggests that the dimerlike defects are mostly magnetic [45]. Figures 5(c) and 5(d) show the tunneling spectra along the arrows in the insets of Figs. 5(a) and 5(b), respectively. There is no zero-bias peak at the vortex center. Upon moving away from the defect site, the impurity states decay, while the broad high-energy core-state peaks appear [shaded regions in Figs. 5(c)-5(d)]. These observations apparently suggest a competition between CdGM and impurity states. We note that the pinned vortices always

have a "hole" in their center in the ZBC map, which also indicates that the low-energy core states are strongly suppressed by the magnetic impurity (see also Figs. S5(d)S5(f)]. Nevertheless, we note that, in some cases, the zerobias vortex state recovers a certain distance away from the defect site, as highlighted by the green curve in Fig. 5(d).

\section{DISCUSSION AND CONCLUSION}

Our data represent the cleanest and most robust zero-bias mode observed in vortices so far. The robustness is manifested in the following aspects:

(i) It is always present at zero bias in free vortex cores, regardless of variations in the underlying superconducting gap.

(ii) It can survive to high magnetic field because of the short coherence length. The $\xi$ of $\left(\mathrm{Li}_{1-x} \mathrm{Fe}_{x}\right) \mathrm{OHFeSe}$ is significantly smaller than that of $\mathrm{FeTe}_{x} \mathrm{Se}_{1-x}$ ( 3 nm) [21] and $\mathrm{Bi}_{2} \mathrm{Te}_{3} / \mathrm{NbSe}_{2}$ ( 30 nm) [16], which also means a higher probability to observe multiple free vortices and a lower probability of pinning by impurities during vortex motion. These points are critical for braiding and fabricating qubits.

(iii) The large superconducting gap and high $T_{c}$ make the system robust against temperature fluctuations and relatively easy to study in experiments.

The cleanness is manifested in three aspects:

(i) The ZBCP is unambiguously separated from other low-lying CdGM states and free from impurity effects in free vortices.

(ii) The FeSe layer is intrinsically stoichiometric, so an improved sample quality will allow more free vortices in larger defect-free regions, enabling further manipulation of the MZMs.

(iii) The width of the ZBCP is the narrowest of all the core states.

The proximity effect of bulk superconductivity induces topological superconductivity in chiral topological surface states [1-3,15], which naturally generates a MZM in the vortex core and explains our observation. Nevertheless, in Sec. S10 of Ref. [30], we use a two-band model to simulate the vortex states with a variety of possible relevant pairing functions (fully gapped to be consistent with our experiment) of the bulk superconducting state, such as pure $s$-wave, $d+i d^{\prime}$, and nodeless $d$-wave, in both the presence and absence of SOC. None of these scenarios would lead to a robust $\mathrm{ZBCP}$. Other origins may lead to a $\mathrm{ZBCP}$ in the tunneling spectrum such as the Kondo effect; SIS tunneling can also be excluded since there is no impurity in the free vortex and the tip used here is nonsuperconducting. Therefore, so far MZM is the most likely origin of the observed clean and robust $\mathrm{ZBCP}$.

To summarize, our experimental and theoretical findings compellingly demonstrate that $\left(\mathrm{Li}_{0.84} \mathrm{Fe}_{0.16}\right) \mathrm{OHFeSe}$, a heavily electron-doped FeSe-based superconductor, is topologically nontrivial. We present the cleanest and most 
robust zero-energy modes in vortices so far, which enable us to obtain the important properties of MZMs with unprecedented accuracy and reliability, including their spatial distribution, scattering rate, response to magnetic impurities, and tunneling quantum efficiency as compared to conventional CdGM states, which would put strong constraints on theory. Our work thus presents an ideal and practical platform to further study the properties of MZMs, explore their manipulation such as braiding, and construct MZM-based qubits, which opens a new, clear route to rapid progress in both the fundamental understanding and potential applications.

\section{ACKNOWLEDGMENTS}

We thank Professors Fuchun Zhang, Jiangping Hu, Jing Wang, Dunghai Lee, and Darren Peets for helpful discussions. This work is supported by the National Natural Science Foundation of China, National Key R\&D Program of the MOST of China (Grants No. 2016YFA0300200, No. 2017YFA0303004, No. 2017YFA0303104, No. 2016YFA0302300, and No. 2017YFA0303003), National Basic Research Program of China (973 Program) under Grant No. 2015CB921700, Science Challenge Project (Grant No. TZ2016004), the Strategic Priority Research Program and Key Research Program of Frontier Sciences of the CAS (Grant No. QYZDY-SSWSLH001), and the Fundamental Research Funds for the Central Universities (Grant No. 310421113). For the calculations, we used high-performance computing clusters at Beijing Normal University in Zhuhai and the National Supercomputer Center in Guangzhou.

Q. L. and C.C. contributed equally.

\section{APPENDIX: EXPERIMENTS AND METHODS}

The high-quality single-crystalline superconducting films of $\left(\mathrm{Li}_{0.84} \mathrm{Fe}_{0.16}\right)$ OHFeSe were grown on a $\mathrm{LaAlO}_{3}$ substrate by a matrix-assisted hydrothermal epitaxial method, as described in Refs. [31,32]. The full widths at half maximum (FWHM) of their x-ray rocking curves are $0.1-0.12$ degrees, indicative of their high quality. The thicknesses of different films vary from 100 to $400 \mathrm{~nm}$.

STM measurements were conducted in a UNISOKU cryogenic STM at $T=0.4 \mathrm{~K}$ or $4.2 \mathrm{~K}$. The sample was cleaved at $78 \mathrm{~K}$ in ultrahigh vacuum with a base pressure of $5 \times 10^{-11}$ Torr and immediately transferred into the STM module. Pt-Ir tips were used after being treated on a clean $\mathrm{Au}$ (111) substrate. The $d I / d V$ spectra were collected by a standard lock-in technique with a modulation frequency of $741 \mathrm{~Hz}$ and a typical modulation amplitude $\Delta V$ of $0.1 \mathrm{mV}$ at $0.4 \mathrm{~K}$ and $1.0 \mathrm{mV}$ at $4.2 \mathrm{~K}$.

ARPES data were taken under an ultrahigh vacuum of $5 \times 10^{-11}$ mbar, with a Fermi Instruments discharge lamp (21.2 eV He-I $\alpha$ light) and a Scienta DA30 electron analyzer. The energy resolution is $6 \mathrm{meV}$, and the angular resolution is $0.3^{\circ}$.

We used fully charged, self-consistent, density functional theory combined with dynamical mean-field theory (DFT + DMFT) [46] to calculate the electronic structure of (Li, Fe)OHFeSe in the paramagnetic state. The DFT part is based on the linearized, augmented, plane-wave method as implemented in WIEN2K [47]. A Hubbard $U$ of $5.0 \mathrm{eV}$ and Hund's coupling $J=0.8 \mathrm{eV}$ were used in the calculations, consistent with previous calculations [48]. The DMFT quantum impurity problem was solved using the continuous-time quantum Monte Carlo method [49] at temperature $T=116 \mathrm{~K}$. We used the experimentally determined crystal structure including the internal atomic positions in our calculations [50]. The surface states were calculated through the iterative Green's function method [51], taking into account the renormalization and shifting of the DFT bands due to strong electronic correlation effects. More specific details of the calculation are described in Sec. S1 of Ref. [30].

[1] X. L. Qi and S. C. Zhang, Topological Insulators and Superconductors, Rev. Mod. Phys. 83, 1057 (2011).

[2] J. Alicea, New Directions in the Pursuit of Majorana Fermions in Solid State Systems, Rep. Prog. Phys. 75, 076501 (2012).

[3] C. W. J. Beenakker, Search for Majorana Fermions in Superconductors, Annu. Rev. Condens. Matter Phys. 4, 113 (2013).

[4] S. D. Sarma, M. Freedman, and C. Nayak, Topologically Protected Qubits from a Possible Non-Abelian Fractional Quantum Hall State, Phys. Rev. Lett. 94, 166802 (2005).

[5] C. Nayak, S. H. Simon, A. Stern, M. Freedman, and S. D. Sarma Non-Abelian Anyons and Topological Quantum Computation, Rev. Mod. Phys. 80, 1083 (2008).

[6] V. Mourik, K. Zuo, S. M. Frolov, S. R. Plissard, E. P. A. M. Bakkers, and L. P. Kouwenhoven Signatures of Majorana Fermions in Hybrid Superconductor-Semiconductor Nanowire Devices, Science 336, 1003 (2012).

[7] A. Das, Y. Ronen, Y. Most, Y. Oreg, M. Heiblum, and H. Shtrikman Zero-Bias Peaks and Splitting in an Al - InAs Nanowire Topological Superconductor as a Signature of Majorana Fermions, Nat. Phys. 8, 887 (2012).

[8] S. Nadj-Perge, I. K. Drozdov, J. Li, H. Chen, S. Jeon, J. Seo, A. H. MacDonald, B. A. Bernevig, and A. Yazdani Observation of Majorana Fermions in Ferromagnetic Atomic Chains on a Superconductor, Science 346, 602 (2014).

[9] H. Zhang et al. Quantized Majorana Conductance, Nature (London) 556, 74 (2018).

[10] J. Alicea, Y. Oreg, G. Refael, F. von Oppen, and M. P. A. Fisher Non-Abelian Statistics and Topological Quantum Information Processing in 1DWire Networks, Nat. Phys. 7, 412 (2011).

[11] S. Vaitiekènas et al. Selective Area Grown SemiconductorSuperconductor Hybrids: A Basis for Topological Networks, Phys. Rev. Lett. 121, 147701 (2018). 
[12] F. Krizek et al. Field Effect Enhancement in Buffered Quantum Nanowire Networks, Phys. Rev. Mater. 2, 093401 (2018).

[13] C. Caroli, P. G. de Gennes, and J. Matricon, Bound Fermion States on a Vortex Line in a Type II Superconductor, J. Phys. Lett. 9, 307 (1964).

[14] G. E. Volovik, Fermions on Quantized Vortices in Superfluids and Superconductors, Turk. J. Phys. 20, 693 (1996).

[15] L. Fu and C. L. Kane, Superconducting Proximity Effect and Majorana Fermions at the Surface of a Topological Insulator, Phys. Rev. Lett. 100, 096407 (2008).

[16] J. P. Xu et al. Experimental Detection of a Majorana Mode in the Core of a Magnetic Vortex Inside a Topological Insulator-Superconductor $\mathrm{Bi}_{2} \mathrm{Te}_{3} / \mathrm{NbSe}_{2}$ Heterostructure, Phys. Rev. Lett. 114, 017001 (2015).

[17] H. H. Sun et al. Majorana Zero Mode Detected with Spin Selective Andreev Reflection in the Vortex of a Topological Superconductor, Phys. Rev. Lett. 116, 257003 (2016).

[18] M. Y. Chen, X. Chen, H. Yang, Z. Du, and H.-H. Wen Superconductivity with Twofold Symmetry in $\mathrm{Bi}_{2} \mathrm{Te}_{3} /$ $\mathrm{FeTe}_{0.55} \mathrm{Se}_{0.45}$ Heterostructures, Sci. Adv. 4, eaat1084 (2018).

[19] S. Sasaki, M. Kriener, K. Segawa, K. Yada, Y. Tanaka, M. Sato, and Y. Ando Topological Superconductivity in $\mathrm{Cu}_{x} \mathrm{Bi}_{2} \mathrm{Se}_{3}$. Phys. Rev. Lett. 107, 217001 (2011).

[20] R. Tao, Y.-J. Yan, X. Liu, Z.-W. Wang, Y. Ando, Q.-H. Wang, T. Zhang, and D.-L. Feng Direct Visualization of the Nematic Superconductivity in $\mathrm{Cu}_{x} \mathrm{Bi}_{2} \mathrm{Se}_{3}$, Phys. Rev. X 8, 041024 (2018).

[21] D. F. Wang et al., Evidence for Majorana bound states in an iron-based superconductor, Science 362, 333 (2018).

[22] P. Zhang et al. Observation of Topological Superconductivity on the Surface of an Iron-based Superconductor, Science 360, 182 (2018).

[23] J. X. Yin et al. Observation of a Robust Zero-Energy Bound State in Iron-based Superconductor $\mathrm{Fe}(\mathrm{Te}, \mathrm{Se})$, Nat. Phys. 11, 543 (2015).

[24] M. Y. Chen, X. Chen, H. Yang, Z. Du, X. Zhu, E. Wang, and H.-H. Wen Discrete Energy Levels of Caroli-de GennesMatricon States in Quantum Limit in $\mathrm{FeTe}_{0.55} \mathrm{Se}_{0.45}$, Nat. Commun. 9, 970 (2018).

[25] N. Hao and J. Hu, Topological Phase in the Single-Layer FeSe, Phys. Rev. X 4, 031053 (2014).

[26] Z. Wang et al. Topological Nature of the $\mathrm{FeSe}_{0.5} \mathrm{Te}_{0.5}$ Superconductor, Phys. Rev. B 92, 115119 (2015).

[27] G. Xu, B. Lian, P. Tang, X.-L. Qi, and S.-C. Zhang Topological Superconductivity on the Surface of Fe-based Superconductors, Phys. Rev. Lett. 117, 047001 (2016).

[28] X. H. Niu et al. Surface Electronic Structure and Isotropic Superconducting Gap in $\left(\mathrm{Li}_{0.8} \mathrm{Fe}_{0.2}\right) \mathrm{OHFeSe}$, Phys. Rev. B 92, 060504(R) (2015).

[29] L. Zhao et al. Common Electronic Origin of Superconductivity in $(\mathrm{Li}, \mathrm{Fe}) \mathrm{OHFeSe}$ Bulk Superconductor and SingleLayer $\mathrm{FeSe} / \mathrm{SrTiO}_{3}$ Films, Nat. Commun. 7, 10608 (2016).

[30] See Supplemental Material at http://link.aps.org/ supplemental/10.1103/PhysRevX.8.041056 for more details about the experiment, band calculation and additional data.
[31] Y. L. Huang et al. Superconducting (Li, Fe)OHFeSe Film of High Quality and High Critical Parameters, Chin. Phys. Lett. 34, 077404 (2017).

[32] Y. L. Huang et al. Matrix-Assisted Fabrication and Exotic Charge Mobility of (Li, Fe)OHFeSe Superconductor Films, arXiv:1711.02920.

[33] M. Yi et al. Observation of Universal Strong OrbitalDependent Correlation Effects in Iron Chalcogenides, Nat. Commun. 6, 7777 (2015).

[34] M. Q. Ren, Y. Yan, X. Niu, R. Tao, D. Hu, R. Peng, B. Xie, J. Zhao, T. Zhang, and D.-L. Feng Superconductivity across Lifshitz Transition and Anomalous Insulating State in Surface $\mathrm{K}$-dosed $\left(\mathrm{Li}_{0.8} \mathrm{Fe}_{0.2} \mathrm{OH}\right) \mathrm{FeSe}$, Sci. Adv. 3, e1603238 (2017).

[35] Y. J. Yan et al. Surface Electronic Structure and Evidence of Plain s-Wave Superconductivity in $\left(\mathrm{Li}_{0.8} \mathrm{Fe}_{0.2}\right)$ OHFeSe, Phys. Rev. B 94, 134502 (2016).

[36] Z. Y. Du, X. Yang, H. Lin, D. Fang, G. Du, J. Xing, H. Yang, X. Zhu, and H.-H. Wen Scrutinizing the Double Superconducting Gaps and Strong Coupling Pairing in $\left(\mathrm{Li}_{1-x} \mathrm{Fe}_{x}\right)$ OHFeSe, Nat. Commun. 7, 10565 (2016).

[37] N. Hayashi, T. Isoshima, M. Ichioka, and K. Machida, Low-Lying Quasiparticle Excitations around a Vortex Core in Quantum Limit, Phys. Rev. Lett. 80, 2921 (1998).

[38] H. F. Hess, R. B. Robinson, R. C. Dynes, J. M. Valles, and J. V. Waszczak Scanning-Tunneling-Microscope Observation of the Abrikosov Flux Lattice and the Density of States Near and Inside a Fluxoid, Phys. Rev. Lett. 62, 214 (1989).

[39] F. Gygi and M. Schlüter, Self-consistent Electronic Structure of a Vortex Line in a Type-II Superconductor, Phys. Rev. B 43, 7609 (1991).

[40] J. Sau, R. M. Lutchyn, S. Tewari, and S. D. Sarma Robustness of Majorana Fermions in Proximity-induced Superconductors, Phys. Rev. B 82, 094522 (2010).

[41] L. Shan et al. Observation of Ordered Vortices with Andreev Bound States in $\mathrm{Ba}_{0.6} \mathrm{~K}_{0.4} \mathrm{Fe}_{2} \mathrm{As}_{2}$, Nat. Phys. 7, 325 (2011).

[42] T. Hanaguri, K. Kitagawa, K. Matsubayashi, Y. Mazaki, Y. Uwatoko, and H. Takagi Scanning Tunneling Microscopy/ Spectroscopy of Vortices in Li, FeAs, Phys. Rev. B 85, 214505 (2012).

[43] Y.E. Kraus, A. Auerbach, H. A. Fertig, and S. H. Simon Testing for Majorana Zero Modes in a $\mathrm{p}_{x}+\mathrm{ip}_{y}$ Superconductor at High Temperature by Tunneling Spectroscopy, Phys. Rev. Lett. 101, 267002 (2008).

[44] L. H. Hu, C. Li, D.-H. Xu, Y. Zhou, and F.-C. Zhang Theory of Spin-Selective Andreev Reflection in the Vortex Core of a Topological Superconductor, Phys. Rev. B 94, 224501 (2016).

[45] W. Li et al. Phase Separation and Magnetic Order in K-doped Iron Selenide Superconductor, Nat. Phys. 8, 126 (2012).

[46] G. Kotliar, S. Y. Savrasov, K. Haule, V. S. Oudovenko, O. Parcollet, and C. A. Marianetti Electronic Structure Calculations with Dynamical Mean-Field Theory, Rev. Mod. Phys. 78, 865 (2006).

[47] P. Blaha, K. Schwarz, G. Madsen, D. Kvasnicka, and J. Luitz, WIEN2K, An Augmented Plane Wave+Local Orbitals Program for Calculating Crystal Properties. 
(Karlheinz Schwarz, Techn. Universität Wien, Austria, 2001).

[48] Z. P. Yin, K. Haule, and G. Kotliar, Kinetic Frustration and the Nature of the Magnetic and Paramagnetic States in Iron Pnictides and Iron Chalcogenides, Nat. Mater. 10, 932 (2011).

[49] K. Haule, Quantum Monte Carlo Impurity Solver for Cluster DMFT and Electronic Structure Calculations in Adjustable Base, Phys. Rev. B 75, 155113 (2007).
[50] X. F. Lu et al. Coexistence of Superconductivity and Antiferromagnetism in $\left(\mathrm{Li}_{0.8} \mathrm{Fe}_{0.2}\right) \mathrm{OHFeSe}$, Nat. Mater. 14, 325 (2015).

[51] Q. S. Wu, S. N. Zhang, H.-F. Song, M. Troyer, and A. A. Soluyanov Wannier Tools: An Open-Source Software Package for Novel Topological Materials, Comput. Phys. Commun. 224, 405 (2018). 